\title{
FATORES ASSOCIADOS À QUALIDADE DE VIDA APÓS MENOPAUSA
}

Dino Roberto Soares De Lorenzi*, Edmund Chada Baracat, Bruno Saciloto, Irineu Padilha Jr.

Trabalho realizado no Ambulatório de Climatério da Universidade de Caxias do Sul, Caxias do Sul, RS

\section{*Correspondência:}

Rua Bento Gonçalves, 1759/602

95020412, Caxias do Sul,

RS, Brasil

dlorenzi@terra.com.br

\begin{abstract}
RESUMO
OBjetivo. Avaliar a qualidade de vida de mulheres na pós-menopausa.

Métodos. Estudo transversal de 323 mulheres pós-menopáusicas com idade entre 45 e 60 anos atendidas em um serviço universitário de atenção ao climatério entre junho e outubro de 2002. A qualidade de vida foi avaliada através do Women's Health Questionnaire. Na análise estatística, utilizou-se o teste t de Student e a análise de variância, seguidos de regressão linear múltipla.

Resultados. A qualidade de vida se mostrou comprometida entre a população estudada, em especial nos domínios relacionados a sintomas somáticos, humor deprimido e ansiedade. Por meio de análise multivariada, constatou-se que quanto menor a escolaridade $(p<0,01)$ e a frequêencia da atividade sexual $(p<0,01)$, assim como a confirmação de comorbidades clínicas prévias $(p=0,03)$, piores os índices de qualidade de vida. Em contrapartida, a atividade física regular se associou aàmelhor qualidade vida $(\mathrm{p}=0,0 \mathrm{I})$. A terapia hormonal, em particular, não se associou à qualidade de vida $(\mathrm{p}=0,48)$.

Conclusã̃o. A qualidade de vida mostrou-se comprometida neste estudo, sendo influenciada tanto por fatores biológicos, quanto por fatores culturais e psicossociais. Possivelmente, as mulheres atribuem à menopausa eventuais sintomas decorrentes de comorbidades clínicas ou dificuldades emocionais prévias, distorcendo a sua percepção acerca desta etapa de suas vidas. Neste sentido, a escolaridade contribuiu para uma maior compreensão das mudanças corporais dessa fase, reduzindo os níveis de ansiedade e estimulando o autocuidado. A sexualidade mostrou-se igualmente um aspecto importante da qualidade de vida no climatério.
\end{abstract}

UnITERMos: Qualidade de vida. Climatério. Menopausa. Terapia hormonal.

\section{INTRODUÇÃO}

O climatério, nas últimas décadas, tem sido reconhecido como mais do que apenas o encerramento da vida reprodutiva feminina. No entanto, ainda que referências a seu respeito encontrem-se descritas em textos escritos por Aristóteles (384-322 a.C.), até recentemente, a condição de mulher "menopausada" era raramente expressa em público, sendo considerada inclusive motivo de constrangimento! .

Em parte, a pouca atenção prestada ao climatério até o início do século passado deveu-se à menor expectativa de vida feminina até então, que não permitia à maioria das mulheres viver o suficiente para atingir o climatério ${ }^{2}$. No entanto, o aumento progressivo da expectativa de vida feminina a partir da segunda metade do século XX, em virtude dos progressivos avanços tecnológicos no campo da saúde, mudou essa situação, desencadeou um interesse crescente pelas questões relacionadas ao envelhecimento feminino ${ }^{3}$.

No Brasil, em 1999, as mulheres com mais de 40 anos correspondiam a 32\% da população feminina do país, com projeção de um aumento ao redor de $11 \%$ até $2010^{5}$. Enquanto, segundo dados censitários, a expectativa de vida feminina no ano 2000 chegava aos 75,6 anos, a masculina não ultrapassava os 64,8 anos ${ }^{5}$.

Do ponto de vista clínico, o climatério é uma etapa marcante do envelhecimento feminino caracterizada pelo estabelecimento de estado fisiológico de hipoestrogenismo progressivo e culminando com a interrupção definitiva dos ciclos menstruais. Inicia-se normalmente entre 35 e 40 anos, estendendo-se até os 65 anos, sendo freqüentemente acompanhado por sintomas característicos e dificuldades na esfera emocional e social ${ }^{6}$.

Nos últimos anos, têm surgido indagações sobre se os sintomas climatéricos e a tendência ao comprometimento da qualidade de vida no climatério, além do hipoestrogenismo, não estariam associados também a fatores psicossociais e culturais relacionados ao processo de envelhecimento ou, então, pela interação destes?

A resposta para estas questões é fundamental para o desenvolvimento de propostas assistenciais voltadas para a mulher climatérica, com vistas a promover um envelhecimento feminino mais sadio e com maior qualidade de vida. Assim, o presente estudo teve por objetivo identificar quais os fatores associados à qualidade de vida em um grupo de mulheres pós-menopáusicas atendidas em um serviço universitário.

\section{Métodos}

Foram entrevistadas 323 mulheres atendidas no Ambulatório de Climatério da Universidade de Caxias do Sul (UCS) entre I de agosto e 31 de outubro de 2002. Os critérios de inclusão foram: mulheres com idade entre 40 e 65 anos, não histerectomizadas e pós-menopáusicas. O estado menopausal foi definido pela história menstrual, tendo-se considerado pós-menopáusica toda mulher em amenorréia espontânea há 12 meses ou mais. Foram excluídas as pacientes que referiram o uso de medicamento não hormonal para o alívio dos sintomas climatéricos nos últimos seis meses ${ }^{8,9}$. 
As variáveis estudadas foram: idade, renda familiar (em salários mínimos per capita), escolaridade (total de anos completos de estudo), cor (definida pela própria paciente como branca ou não branca), ocupação (com ou sem ocupação remunerada), estado marital (com ou sem companheiro fixo), número de relações sexuais no último mês, número de filhos, idade de ocorrência da menopausa, prática de atividade física regular (no mínimo três vezes por semana e por 60 minutos ou mais), tabagismo (consumo diário de cigarros nos últimos seis meses), índice de massa corporal, história de comorbidades clínicas prévias (hipertensão arterial, diabetes, câncer e doença cardiovascular prévia) e terapia hormonal (dicotomizada em sim ou não, conforme a confirmação do seu uso continuado havia pelo menos seis meses, independente do esquema e do tipo de medicação usada) $)^{6,10,11}$.

A qualidade de vida no climatério foi avaliada através do Questionário de Saúde da Mulher (Women's Health Questionnaire). Este instrumento foi previamente validado para a língua portuguesa, sendo composto por 36 itens agrupados em nove domínios: sintomas somáticos, humor deprimido, dificuldades cognitivas, ansiedade, satisfação sexual, sintomas vasomotores, distúrbios do sono, atração e sintomas menstruais (este último não foi avaliado, visto terem sido estudadas somente mulheres pós-menopáusicas). Quanto maior o escore obtido, pior a qualidade de vida ${ }^{12}$.

Os dados coletados foram digitados e analisados eletronicamente por meio do programa Statistical Package for Social Sciences for Personal Computer (SPSS-PC) versão 10. Para a comparação de médias, usou-se o teste $t$ de Student e a análise de variância (Anova). A associaçãa entre as variáveis estudadas e o desfecho (escores de qualidade de vida) foi avaliada por meio de regressão linear múltipla. A fim de viabilizar a sua inclusão no modelo de regressão, as variáveis categóricas (tabagismo, estado marital, cor, ocupação, prática regular de exercícios físicos, história de comorbidades clínicas e uso de terapia hormonal) foram transformadas em variáveis dummy ${ }^{13}$. Em todas as análises realizadas, aceitou-se um nível de significância de 5\%.

Este estudo foi submetido previamente à apreciação do Comitê de Ética em Pesquisa da Universidade de Caxias do Sul, tendo sido aprovado. Antes da inclusão de qualquer paciente, esta foi esclarecida acerca da sua metodologia, objetivos e possíveis riscos, assinando após um termo de consentimento livre e esclarecido.

\section{Resultados}

A idade média das mulheres estudadas foi de 53,7 ( \pm 4, I) anos, sendo $70,9 \%$ de cor branca. Cerca de $54,5 \%$ referiram menos de cinco anos completos de estudo, sendo 9,9\% analfabetas. A renda familiar média foi de I,8 ( \pm I, I) salário mínimo per capita, sendo que 32,8\% das entrevistadas confirmaram uma ocupação remunerada (Tabela I).

A maioria das pacientes $(63,8 \%)$ referiu um companheiro fixo, sendo $60,7 \%$ sexualmente ativas. Somente $6,2 \%$ negaram ter tido filhos (Tabela I). A menarca ocorreu, em média, aos 13,2 ( \pm 1,9) anos e a menopausa aos 47,7 anos $( \pm 4,5)$. Tabagismo foi referido por $28,5 \%$ das mulheres avaliadas, que apresentaram taxas de sobrepeso e obesidade $\left(I M C \geq 25 \mathrm{Kg} / \mathrm{m}^{2}\right)$ de $61,3 \%$. Somente $21,4 \%$ das

\section{Tabela I - Escores médios de qualidade de vida} segundo variáveis sociodemográficas

\begin{tabular}{|c|c|c|c|}
\hline & $n(\%)$ & $\begin{array}{c}\text { Escore de qualidade de vida } \\
\text { Média (DP) }\end{array}$ & $p$ \\
\hline $\begin{array}{l}\text { Idade (em anos) } \\
\quad 45-49 \\
50-54 \\
55-60 \\
\text { Média(DP) }\end{array}$ & $\begin{array}{c}60(18,6) \\
120(37,2) \\
43(44,3) \\
53,7(4,1)\end{array}$ & $\begin{array}{l}79,4(19,7) \\
79,3(19,2) \\
75,8(19,1)\end{array}$ & $0,19(2)$ \\
\hline Cor & & & \\
\hline $\begin{array}{l}\text { Branca } \\
\text { Não branca }\end{array}$ & $\begin{array}{l}229(70,9) \\
94(29,1)\end{array}$ & $\begin{array}{l}74,1(18,3) \\
79,4(20,1)\end{array}$ & 0,03 \\
\hline
\end{tabular}

Escolaridade

(anos completos

de estudo)

\begin{tabular}{llll}
$<5$ & $2176(54,5)$ & $79,3(18,6)$ & \\
$5-9$ & $103(31,9)$ & $73,8(\mid 8,9)$ & $<0,0 \mid(2)$ \\
$>10$ & $44(13,6)$ & $65,3(18,5)$ & \\
\hline Média(DP) & $5,0(2,6)$ & & \\
\hline
\end{tabular}

\begin{tabular}{llll}
\hline $\begin{array}{l}\text { Ocupação } \\
\text { remunerada }\end{array}$ & & & \\
$\quad$ Sim & $106(32,8)$ & $74,3(19,5)$ & $0,38(1)$ \\
$\quad$ Não & $217(67,2)$ & $74,2(18,6)$ & \\
\hline $\begin{array}{l}\text { Renda familiar } \\
\text { (salários mínimos }\end{array}$ & & & \\
$\begin{array}{l}\text { per capita) } \\
\quad<1\end{array}$ & $17 \mid(52,9)$ & $79,5(18,3)$ & \\
$\quad$ I-2 & $100(31,0)$ & $73,7(18,2)$ & $0,03(2)$ \\
$\geq 3$ & $52(16,1)$ & $66,8(20,9)$ & \\
Média(DP) & $1,8(1,1)$ & & \\
\hline Estado marital & & & \\
$\quad$ Com companheirofixo & $206(63,8)$ & $75,8(19,1)$ & $0,82(1)$ \\
$\quad$ Sem companheirofixo & $117(36,2)$ & $75,3(19,6)$ & \\
\hline Total & $323(100)$ & $75,6(19,2)$ & \\
\hline
\end{tabular}

(1) Teste t de Student ${ }^{(2)}$ Anova

Teste de Comparações Múltiplas de Tukey - Constatou-se diferenças significativas nos escores de qualidade de vida entre todos grupos relativos à escolaridade. Quanto à renda familiar, observaramse diferenças significativas entre as mulheres que recebiam menos de um salário mínimo per capita e as que possuíam uma renda familiar per capita entre um e dois salários e as com uma renda de três ou mais salários mínimos per capita, não havendo diferenças significativas entre estes dois últimos

entrevistadas confirmaram atividade física regular. Além disso, a maioria $(64,4 \%)$ confirmou comorbidades clínicas prévias: história de câncer $(n=18 / 5,6 \%)$, hipertensão arterial sistêmica $(n=160 / 49,5 \%)$, diabetes $(n=56 / 17,3 \%)$ e infarto agudo do miocárdio $(n=24,7,4 \%)$ (Tabela 2).

A análise do Questionário de Saúde da Mulher revelou um escore médio de qualidade de vida de 75,6 $( \pm 19,2)$. Os valores dos diferentes domínios que compõem este instrumento, em ordem decrescente de comprometimento, foram: sintomas somáticos $(17,5 / \pm 5,3)$, humor deprimido ( $12,8 / \pm 4,8)$, ansiedade $(8,7 / \pm 3, I)$, problemas com o sono $(8,1 / \pm 2,8)$, sintomas vasomotores $(6,5 / \pm 2,7)$, dificuldades na esfera sexual $(6,3 / \pm 2,5)$, alteração da capacidade de atração $(5,6 / \pm 2,3)$ e dificuldades cognitivas $(4,5 / \pm 2,2)$.

Não se verificou qualquer associação entre os níveis de qualidade de vida e a variável idade, estado marital, ocupação, idade da 


\section{Tabela 2 - Escores de qualidade de vida segundo variáveis} reprodutivas, condições de saúde e estilo de vida

\begin{tabular}{|c|c|c|c|}
\hline & $\mathrm{n}(\%)$ & $\begin{array}{l}\text { Escore de qualidade de vida } \\
\text { Média (DP) }\end{array}$ & $p$ \\
\hline $\begin{array}{l}\text { Idade da menopausa } \\
\text { (em anos) } \\
\quad<45 \\
45-49 \\
\geq 50 \\
\text { Média(DP) }\end{array}$ & $\begin{array}{l}59(18,3) \\
140(43,3) \\
124(38,4) \\
47,7(4,5)\end{array}$ & $\begin{array}{l}79,4(19,7) \\
73,9(19,2) \\
75,8(19,1)\end{array}$ & $0,19^{(2)}$ \\
\hline $\begin{array}{l}\text { Número de filhos } \\
\text { Semfilhos } \\
\text { I a } 3 \\
>3 \\
\text { Média(DP) }\end{array}$ & $\begin{array}{c}20(6,2) \\
172(53,3) \\
13 \mid(40,6) \\
3,5(1,7)\end{array}$ & $\begin{array}{l}75,2(21,5) \\
73,4(19,1) \\
78,6(18,8)\end{array}$ & $0,66^{(2)}$ \\
\hline
\end{tabular}

\begin{tabular}{llll}
\hline $\begin{array}{l}\text { Relações sexuais no } \\
\text { último mês }\end{array}$ & & & \\
$\quad$ Sim & $196(60,7)$ & $74,3(18,9)$ & $0,04(1)$ \\
Não & $127(39,3)$ & $77,8(19,6)$ & \\
Média(DP) & $3,8(2,3)$ & & \\
\hline Tabagismo & & & \\
$\quad$ Sim & $92(28,5)$ & $76,6(18,8)$ & 0,57 (1) \\
Não & $231(71,5)$ & $75,3(19,4)$ & \\
\hline Comorbidades clínicas & & & \\
$\quad$ Sim & $208(64,4)$ & $77,5(19,5)$ & $0,82^{(1)}$ \\
Não & $115(35,6)$ & $72,3(18,3)$ & \\
\hline Terapia hormonal & & & \\
Sim & $69(21,4)$ & $74,1(16,4)$ & 0,45 (1) \\
Não & $254(78,6)$ & $76,1(19,9)$ & \\
\hline
\end{tabular}

\begin{tabular}{llll}
\hline $\begin{array}{l}\text { Prática regular de } \\
\text { exercíciosfísicos }\end{array}$ & & & \\
Sim & $94(21,4)$ & $69,6(19,2)$ & $<0,01(1)$ \\
Não & $229(78,6)$ & $78,1(18,7)$ & \\
\hline
\end{tabular}

\begin{tabular}{llll}
\hline Índice de massa & & & \\
corporal $\left(\mathrm{Kg} / \mathbf{m}^{2}\right)$ & & & \\
$\geq 25(\mathrm{com}$ & & & \\
sobrepeso/obesidade) & $198(61,3)$ & $76,7(19,8)$ & 0,18 (1) \\
$\quad<25($ sem & & & \\
sobrepeso/obesidade) & $125(38,7)$ & $73,9(18,2)$ & \\
$\quad$ Média(DP) & $27,2(5,4)$ & & \\
\hline Total & $323(100)$ & $75,6(19,2)$ & \\
\hline
\end{tabular}

(I) Teste t de Student (2) Anova

menopausa, número de filhos, tabagismo e índice de massa corporal (Tabelas 2 e 3). Em contrapartida, a qualidade de vida foi significativamente pior entre as mulheres não brancas $(p=0,03)$, com baixa escolaridade $(p<0,0 \mathrm{I})$, menor renda familiar per capita $(p=0,03)$, sem atividade física regular $(p<0,01)$ e com comorbidades clínicas prévias $(p=0,02)$. A terapia hormonal, em especial, não se associou à qualidade de vida $(p=0,45)$, ao passo que a confirmação de atividade sexual implicou em maior qualidade de vida $(p=0,04)$ (Tabela 2).

Por meio de análise multivariada, mostraram-se fatores associados à qualidade de vida neste estudo: a escolaridade $(p<0,01)$, a freqüência da atividade sexual $(p<0,0 I)$, a atividade física $(p=0,0 I)$ e a história de
Tabela 3 - Fatores associados à qualidade de vida entre mulheres pós-menopáusicas: análise por regressão linear múltipla

\begin{tabular}{lcc}
\hline Variáveis & $\boldsymbol{\beta}$ & $\frac{\boldsymbol{p}}{<0,01}$ \\
Escolaridade (anos completos de estudo) & $-0,22$ & $<0,01$ \\
$\begin{array}{l}\text { Freqüência da atividadade sexual } \\
\text { (número de relaçôes sexuais no último mês) }\end{array}$ & $-0,23$ & $<0,01$ \\
Prática regular de exerćicios físicos & $-0,14$ & 0,01 \\
Comorbidades clínicas & 0,12 & 0,03 \\
\hline
\end{tabular}

Análise ajustada para: idade, escolaridade, cor, ocupação, rendafamiliar per capita, estado martal, número de filhos, número de relações sexuais no úttimo mês, tabagismo, história de co-morbidades clínicas, idade da menopausa, uso de terapia hormonal, prática regular de exercícios, índice de massa corporal

comorbidades clínicas $(p=0,03)$. Assim, quanto menos anos completos de estudo, menor o número de relações sexuais no mês anterior à entrevista e a história de comorbidades clínicas prévias associaram-se à pior qualidade de vida. A confirmação, por sua vez, de atividade física regular se associou a melhores escores de qualidade vida (Tabela3).

\section{Discussão}

A crescente preocupação atual com a qualidade de vida no campo das ciências biomédicas, em parte, originou-se nos progressivos avanços ocorridos a partir da segunda metade do século passado, que trouxeram aos profissionais de saúde não somente a necessidade de um maior conhecimento acerca dos sentimentos e percepções dos pacientes sobre as suas condições de saúde, como de mensurar o impacto de eventuais intervenções terapêuticas na sua vida e bem-estar ${ }^{14}$.

Neste estudo, a qualidade de vida das mulheres pesquisadas, com base no Questionário de Saúde da Mulher, mostrou-se comprometida. $O$ escore global de qualidade de vida atingiu o valor de 75,6 ( $\pm 19,2)$. Os domínios mais comprometidos foram os sintomas somáticos, humor deprimido, ansiedade, distúrbios do sono e sintomas vasomotores, achados estes semelhantes ao observado em estudo similar realizado no Setor de Climatério da Universidade Federal de São Paulo'2.

Estima-se que $50 \%$ a $70 \%$ das mulheres climatéricas manifestem sintomas somáticos e dificuldades emocionais ${ }^{15}$. Cerca de $78,6 \%$ das mulheres pesquisadas eram sedentárias, com uma taxa de sobrepeso e obesidade de $61,3 \%$, o que pode ter interferido negativamente na sua qualidade de vida. Além disso, 64,4\% confirmaram comorbidades clínicas prévias, o que pode explicar muitas das queixas somáticas e dificuldades emocionais observadas ${ }^{16}$.

Quanto à esfera emocional, o estradiol modularia neurotransmissores químicos, interferindo no humor. O hipoestrogenismo reduziria a secreção de endorfinas cerebrais, favorecendo a depressão, dificuldades cognitivas e até processos demenciais. A labilidade emocional após a menopausa estaria relacionada também à severidade das ondas de calor, visto estas interferirem no sono, causando irritabilidade e dificuldades no convívio social"17.

A maior tendência à depressão no climatério tem sido atribuída também ao medo de envelhecer e à percepção de proximidade da morte, sentimentos estes agravados pela sensação de inutilidade ou carência afetiva. O climatério é uma fase de transição física e social, coincidindo com a independência dos filhos, a morte de familiares 
e a aposentadoria, circunstâncias estas que requerem ajustes emocionais difíceis para a mulher ${ }^{18}$.

As reações emocionais no climatério parecem ser particularmente influenciadas pelas atitudes em relação à menopausa, sendo menos intensas entre as mulheres que associam a menopausa à maior maturidade e autoconfiança. Todavia, para as mulheres que conseguem redimensionar novas perspectivas existenciais, o climatério é acompanhado de sintomas mais intensos, maior irritabilidade, ansiedade, depressão e até dificuldades sexuais ${ }^{19}$.

Insatisfação com o sono foi evidente neste estudo. Pesquisas mostram que, ao contrário das demais queixas climatéricas que tendem a melhorar com o passar do tempo, as dificuldades com o sono parecem se intensificar nos anos que se seguem à menopausa. Contudo, ainda não foi possível estabelecer uma relação definitiva entre os distúrbios do sono e as oscilações esteroídicas que caracterizam essa fase, podendo estar realcionadas à severidade dos sintomas vasomotores ou a transtornos psíquicos ${ }^{20}$.

$\mathrm{Na}$ última década, têm sido descritas discordâncias na percepção acerca da qualidade do sono pelas mulheres no climatério e os resultados dos exames polissonográficos, o que talvez se deva à maior prevalência de sintomas somáticos e emocionais nessa fase, o que poderia distorcer a sua percepção sobre a qualidade de seu sono ${ }^{2 !}$.

Neste estudo, os sintomas vasomotores interferem negativamente na qualidade de vida no climatério, acometendo $18 \%$ a $74 \%$ das mulheres nessa fase. Porém, inclusive estes parecem ser influenciados por fatores psicossociais22.

A análise multivariada mostrou que a qualidade de vida entre as mulheres entrevistadas era influenciada pelos seguintes fatores: escolaridade, história de comorbidades clínicas, atividade física e freqüência da atividade sexual.

Os sintomas climatéricos parecem ser menos intensos entre as mulheres com maior nível educacional e que se exercitam regularmente ${ }^{23}$. Ao se aproximarem da menopausa, as mulheres trazem dúvidas sobre as modificações físicas que irão ocorrer e de como lidar com elas. A maior escolaridade não apenas facilita o acesso à informação sobre o climatério, como reduz a ansiedade comum nessa fase ${ }^{24}$. O próprio autocuidado é influenciado pela escolaridade; pesquisa realizada em Cuba revelou que mulheres com maior nível educacional procuram assistência médica com uma freqüência 2,7 vezes maior ${ }^{25}$.

A atividade física regular contribuiu para a preservação da massa muscular e da flexibilidade articular, reduzindo a intensidade dos sintomas somáticos e levando a uma sensação de maior bem-estar no climatério ${ }^{24}$. 0 exercício físico não somente aumenta a secreção de b-endorfinas hipotalâmicas, aliviando as ondas de calor e melhorando o humor, como aumenta a densidade mineral óssea, diminui a frequêencia cardíaca de repouso, melhora o perfil lipídico e normaliza a pressão arterial ${ }^{26}$. Por fim, a atividade física melhora a imagem corporal, aumentando a auto-estima feminina ${ }^{16}$.

A frequiência da atividade sexual influenciou os escores de qualidade de vida neste estudo. As disfunções sexuais, no entanto, acometem $25 \%$ a $33 \%$ das mulheres entre $35-59$ anos e $51 \%$ a $75 \%$ das mulheres entre 60-65 anos. O hipoestrogenismo causa atrofia urogenital, levando a dispareunia e diminuição da atividade sexual ${ }^{27}$. 0 humor depressivo, o envelhecimento físico e a tendência a aumento de peso após a menopausa, por sua vez, afetam a auto-imagem, favorecendo a baixa auto-estima e um menor desejo sexual ${ }^{27,28}$.

Outros autores têm atribuído as dificuldades na esfera sexual a sentimentos de culpa ou constrangimento pelo desejo sexual por parte da mulher ou a eventuais dificuldades conjugais. Além disso, a atividade sexual pode ser afetada por comorbidades pré-existentes da própria mulher ou de seu parceiro que, pelas suas repercussões clínicas ou necessidade de uso de medicamentos, interferem na libido ou na potência sexual29.

Não se constatou associação entre o uso de terapia hormonal e os escores de qualidade de vida nesta casuística. Nesse sentido, a literatura tem se mostrado também controversa. $\mathrm{Na}$ Itália, foi avaliado o efeito da administração diária de $1 \mathrm{mg}$ de 17 betaestradiol e $0,5 \mathrm{mg}$ de acetato de noretisterona na qualidade de vida de mulheres pós-menopáusicas em comparação a um grupo controle sem terapia hormonal. Os resultados deste estudo revelaram um alívio dos sintomas vasomotores, das queixas somáticas, da instabilidade de humor e das dificuldades com o sono entre as usuárias de terapia hormona ${ }^{30}$. Pesquisa norte-americana avaliou os efeitos da terapia estro-progestativa combinada contínua na qualidade de vida de 8506 mulheres pós-menopáusicas norteamericanas em relação a 8102 mulheres sem terapia hormonal. Após três anos de seguimento, não foi possível identificar diferenças significativas nos escores de qualidade de vida entre os dois grupos avaliados ${ }^{3 !}$.

No Brasil, estudo transversal avaliou 207 mulheres pós-menopáusicas, 106 usuárias e 101 não usuárias de terapia hormonal, não tendo identificado também diferenças significativas nos escores de qualidade de vida entre estas.

Possivelmente, os sintomas vasomotores e da atrofia urogenital são os únicos realmente influenciados pelo estado menopausal, ao passo que as demais queixas climatéricas parecem ser influenciadas principalmente por fatores psicossociais, estilo de vida e pelas atitudes da mulher em relação à menopausa ${ }^{32}$.

A influência do estado prévio de saúde na qualidade de vida na pós-menopausa tem merecido destaque, visto que, possivelmente, muitas das queixas referidas nessa fase podem ser decorrentes de comorbidades clínicas prévias ${ }^{10}$. Pesquisa realizada com mulheres cardiopatas isquêmicas em São Paulo não somente revelou que estas apresentavam uma maior limitação física nas atividades cotidianas, como maiores dificuldades emocionais, quando comparadas com um grupo controle sem a doençal 19 .

É importante lembrar que o corrente estudo foi realizado em um serviço especializado de atenção ao climatério, que disponibiliza uma assistência multiprofissional e individualizada, envolvendo atividades de educação em saúde e promoção ao autocuidado, o que pode ter contribuído para uma atitude mais positiva em relação à menopausa, minimizando os efeitos da terapia hormonal". Ainda que os seus achados não possam ser extrapolados para a população em geral, espera-se que estes estimulem novas pesquisas voltadas a aprofundar o conhecimento sobre o impacto do climatério na qualidade de vida da mulher brasileira. A maioria das informações disponíveis acerca deste tema é ainda proveniente de pesquisas conduzidas em outros 
países, cuja extrapolação para a realidade brasileira é limitada por diferenças culturais e socioeconômicas.

\section{Conclusão}

A qualidade de vida mostrou uma tendência ao comprometimento neste estudo, sendo influenciada tanto por fatores biológicos, quanto por fatores culturais e psicossociais. Possivelmente, as mulheres atribuem à menopausa eventuais sintomas decorrentes de comorbidades clínicas ou dificuldades emocionais prévias, o que distorce sua percepção acerca desta etapa de suas vidas. Nesse sentido, a escolaridade contribuiu para uma maior compreensão das mudanças corporais dessa fase, reduzindo os níveis de ansiedade e estimulando o autocuidado. A sexualidade mostrou-se também um aspecto importante da qualidade de vida no período pós-menopausa.

\section{Conflito de interesse: não há}

\section{SUMMARY}

\section{Factors Related to QUALITY OF LIFE IN POST-MENOPAUSE}

OBIECTIVE. To assess quality of life of postmenopausal women.

METHODS. A cross-section study of 323 women between 45 and 60 years of age attended at a university climacteric clinic from June to October 2002was carried out. Quality of life was assessed by the Women's Health Questionnaire (WHQ). Statistical analysis was performed with Student's t Test and analysis of variance, followed by multiple linear regression analysis.

RESULTS. This study found quality of life impaired especially in the domains related to somatic symptoms, depressed mood, and anxiety. Multivariate analysis showed that lower educational level $(p<0.01$, frequency of sexual activity $(p<0.01)$ and the confirmation of previous clinical co-morbidities $(p=0.03)$ were associated to the worst scores of quality of life. On the other hand, regular physical activity was related to better quality of life $(p=0.01)$. Hormone therapy, in particular, was not related to quality of life $(p=0.48)$.

CONCLUSION. Quality of life was found not only to be influenced by biological factors, but also by psychossocial and cultural factors. Middle aged women frequently attributed eventual symptoms associated to clinical co-morbidities or previous emotional difficulties to menopause, distorting their perception of this phase in their life. In this sense, the educational level contributed to a better understanding of body changes at this time, reducing anxiety levels and encouraging self-care. Sexuality was also an important aspect related to quality of life in the climacterium. [Rev Assoc Med Bras 2006; 52(5): 312-7]

KEY WORDS: Quality of life. Climacterium. Menopause. Hormonal therapy.

\section{REFERÊNCIAS}

I. Utian WH. Menopause, a modern perspective from a controversial history. Maturitas 1993;26:73-82.

2. Hardy E, Alves G, Osis MJD. Climatério: implicações sociais. Femina 1992;20:313-20

3. Uchôa E. Contribuições da antropologia para uma abordagem das questões relativas à saúde do idoso. Cad Saúde Públ 2003;3:849-53.

4. Zahar SEV, Aldrighi JM, Tostes MA, Russomano F, Zahar LO. Avaliação da qualidade de vida na menopausa. Reprod Clim; 200 I; | 6: I 63-72.

5. Renó Jr J. Alterações do humor e da cognição: fisiopatologia, diagnóstico e tratamento. In: Fernandes CE, editor. Menopausa e Tratamento. São Paulo: Editora Segmento; 2003. p. I I I-6.

6. De Lorenzi DRS, Danelon C, Saciloto B, Padilha Jr I. Fatores indicadores da sintomatologia climatérica. RBGO 2005; 27: I2-9.

7. Dennerstein L, Lehert P, Guthrie J. The effects of the menopausal transition and biopsychossocial factors on well-being. Arch Women Ment Health 2002; 5: 15-22.

8. Rodrigues de Lima JG, Baracat EC. Síndrome do climatério. In:Rodrigues de Lima JG, Baracat EC. Ginecologia Endócrina. I ${ }^{a}$ ed. São Paulo: Editora Atheneu; 1995. p.253-98.

9. Hunter M. The southeast England longitudinal study of the climacteric and postmenopausal. Maturitas 1992; 14: I 17-26.

10. Thompson PD, Buchner D, Pina IL. Exercise and physical activity in the prevention and treatment of atherosclerotic cardiovascular disease: a statement from the Council on Clinical Cardiology (Subcommittee on Exercise, Rehabilitation, and Prevention) and the Council on Nutrition, Physical Activity, and Metabolism (Subcommittee on Physical Activity). Circulation. 2003; 107:3 109-16.10

I I. Zahar EV, Aldrighi JM, Pinto Neto AM, Conde DM, Zahar LO, Russomano F. Qualidade de vida em usuárias e não usuárias de terapia de reposição hormonal. Rev Assoc Med Bras 2005; 5 I : I 33-8.

12. Silva Filho CR, Baracat EC, Conterno LO, Haidar MA, Ferraz MB. Sintomas climatéricos e qualidade de vida: validação do questionário da saúde da mulher. Rev Saúde Pública 2005; 39:333-9.

13. Zar JH. Biostatistical analysis. New Jersey: Prentice-Hall Inc.; 1984. p.328-33.

14. Cianciarullo TI. Compreendendo a família no cenário de uma nova estratégia de saúde. In: Cianciarullo TI, Gualda DMR, Da Silva GTR, Cunha ICKO. Saúde na família e na comunidade. I ${ }^{a}$ ed. São Paulo: Robes Editoria; 2002. p. 17-37.

15. Hay AG, Banckroft J, Johnstone EC. Affective symptoms in women attending a menopause clinic. British Journal of Psychiatry 1994; 164:513-6.

16. Ferriani RA. Tratamento do climatério: medidas alternativas e estilo de vida. Reprod Clim Supl 200 I ; 6:54-60.

17. Aldrighi JM, Aldrighi CMS, Aldrighi APS. Alterações sistêmicas do climatério. Rev Bras Med 2002; I 5:21.

18. Dennerstein L, Lehert P, Guthrie J. The effects of the menopausal transition and biopsychossocial factors on well being. Arch Women Ment Health 2002; 5: 15-22.

19. Favarato MECS, Aldrighi JM, Fráguas Jr. R, Pires ALR, Lima SMRR. Sexualidade e climatério: influência de fatores biológicos, psicológicos e sócio-culturais. Reprod Clim 200 I; I 5: 199-202.

20. Souza LS, Aldrighi JM. Sono e climatério. Reprod Clim 200 I ; 6:20-5.

2 I. Polo-Kantola P. Climacterics symptoms and sleep quality. Obstet Gynecol 1998:94:219.

22. Sclowitz KTS, Santos IS, Silveira MF. Prevalência e fatores associados a fogachos em mulheres climatéricas e pós-climatéricas. Cad Saúde Pública 2005; 21:109-21. 
23. Mendito A, Cassese E, Balbi C. Climaterio e qualitá della vita. Minerva Ginecol 1999;51:83-9.

24. Liao KKM, Hunter MS. Preparação para a menopausa: avaliação das expectativas de uma intervenção educativa de saúde na mulher de meiaidade. Maturitas-Brasil 1999; 1:59-67.

25. Alpizar ER, Despaigne DN. Factores relacionados con la demanda de atención médica para el climaterio. Rev Cuba Endocrinol [serial on line] 2003 Sept-Dec [cited 2005 Sept 0 I]; I 4(3). Available from: URL:http://bvs.sld.cu/revistas/end/voll 4_3_03303.htm.

26. Bossemeyer RP. Atividade física no climatério. In: Fernandes CE. Menopausa e Tratamento. São Paulo: Editora Segmento, 2003. p. $201-9$.

27. Favarato MECS, Aldrighi JM, Fráguas Jr. R, Pires ALR, Lima SMRR. Sexualidade e climatério: influência de fatores biológicos, psicológicos e sócio-culturais. Reprod Clim 2000; 1 5: 199-202.

28. Chiechi LM, Granieri M, Lobascio A, Ferreri R, Loizzi P. Sexuality in the climacterium. Clin Exp Obst \& Gyn 1997;24: I 58-9.

29. Lauritzen C. Biology of female sexuality in old age. Z Gerontol 1983;16:134.
30. Gambacciani M, Ciaponi M, Cappagli B, Monteleone P, Benussi C, Bevilacqua G, Genazzani AR. Effects of low-dose, continuous combined estradiol and noretisterone acetate on menopausal quality of life in early postmenopausal women. Maturitas. 2003;44: I 57-63.

31. Hays J, Ockene JK, Brunner RL, Kotchen JM, Manson JE,Patterson RE, Aragaki AK, Shumaker SA, Brzyski RG, LaCroixAZ, Granek IA, and Valanis BG. Effects of Estrogen/Progestin on Health-Related Quality of Life. N Engl J Med 2003; 348: | 839-54.

32. Olofsson AS, Collins A. Psychosocial factors, attitude towards menopause and symptoms in Swedish perimenopausa women. Climacteric 2000;3:33-42.

Artigo recebido: 10/09/2005

Aceito para publicação: 03/I I/2005 